Provided for non-commercial research and educational use only. Not for reproduction or distribution or commercial use.

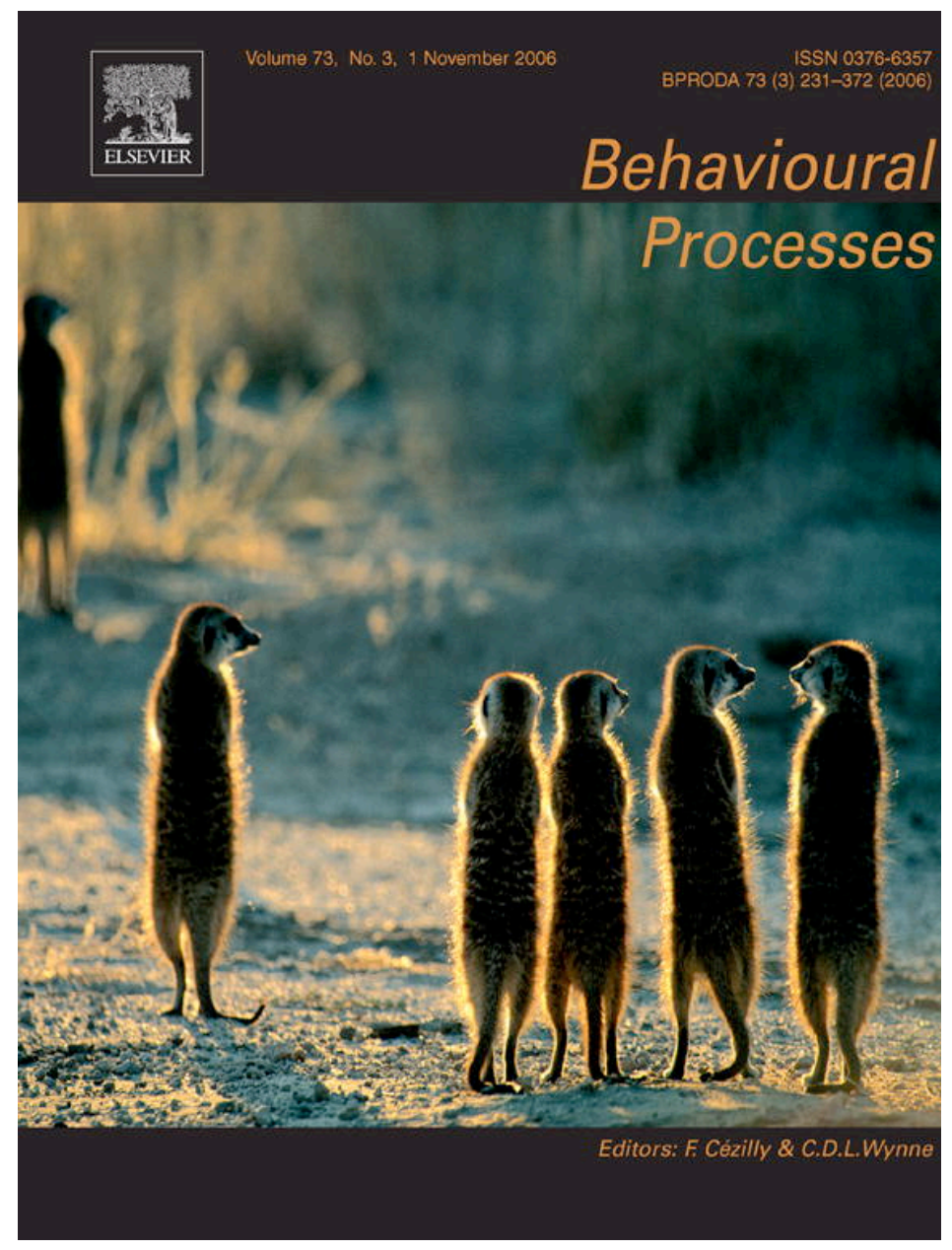

This article was originally published in a journal published by Elsevier, and the attached copy is provided by Elsevier for the author's benefit and for the benefit of the author's institution, for non-commercial research and educational use including without limitation use in instruction at your institution, sending it to specific colleagues that you know, and providing a copy to your institution's administrator.

All other uses, reproduction and distribution, including without limitation commercial reprints, selling or licensing copies or access,

or posting on open internet sites, your personal or institution's website or repository, are prohibited. For exceptions, permission may be sought for such use through Elsevier's permissions site at: 


\title{
Stealing of dunked food in Carib grackles (Quiscalus lugubris)
}

\author{
Julie Morand-Ferron*, Mélisa Veillette, Louis Lefebvre \\ Department of Biology, McGill University, 1205 avenue Docteur Penfield, Montréal, Québec, Canada H3A 1 B1 \\ Received 8 May 2006; received in revised form 11 August 2006; accepted 23 August 2006
}

\begin{abstract}
The use of tool or tool-like food processing behaviours can render animals vulnerable to theft (kleptoparasitism) because (1) large, nutritious items are usually involved, (2) value is added to the food due to long and/or complex handling, and (3) physical control of items is often temporarily lost during handling. In Barbados, Carib grackles (Quiscalus lugubris) immersing items in water before consumption (a behaviour known as food dunking) lose a larger proportion of items to conspecific food thieves than grackles that do not dunk. In this paper, we first show that dunking in Carib grackles functions as a proto-tool food-processing technique that speeds up ingestion. We then examine five potential predictors of kleptoparasitism: only conspecific density and loss of physical control on food were found to influence the probability that birds would be attacked and successfully robbed of food by conspecifics. Grackles could reduce the probability of kleptoparasitism by holding items in the bill while dunking and engaging in head-up displays. These behaviours were used flexibly depending on variation in the risk of kleptoparasitism. We suggest that costs like the ones incurred from theft might limit the profitability and frequency of tool and proto-tool food processing behaviours, creating a context where counter-strategies might be selected.
\end{abstract}

(c) 2006 Elsevier B.V. All rights reserved.

Keywords: Anti-kleptoparasitic tactic; Food processing; Kleptoparasitism; Tool use

Foragers can exploit the searching and handling efforts of others by engaging in food-stealing, or kleptoparasitism (Brockmann and Barnard, 1979). Any situation that lengthens handling or reduces control over high-quality food items may increase the benefits of parasitic tactics in a population (Giraldeau and Caraco, 2000). The use of environmental features to modify or manipulate food might be one of these situations: when a gull drops a shell on a hard substrate (e.g. Norris et al., 2000) or a macaque uses water to separate wheat grains from sand (Kawai, 1965), it becomes vulnerable to exploitation by competitors. This potential "scrounging cost" has not been recognised in explaining the low frequency of tool, proto-tool (Parker and Gibson, 1977), and other food processing behaviours in wild animal populations, although it has been observed previously that some individuals will refrain using a tool or proto-tool in the presence of potential kleptoparasites (e.g. Visalberghi and Fragaszy, 1990). Here, we focus on dunking, a processing technique used by several species of grackles (Jackson, 1985) and corvids (Goodwin, 1986) and examine the factors affecting vulnerability and the counter-tactics of dunkers faced with the risk of intraspecific kleptoparasitism.

\footnotetext{
* Corresponding author. Tel.: +1 514 3986467; fax: +1 5143985069.

E-mail address: julie.morand-ferron@mail.mcgill.ca (J. Morand-Ferron).
}

Our study species is the Carib grackle (Quiscalus lugubris), a generalist passerine that feeds in groups on high-quality, clumped food resources (mostly anthropogenic) in urban areas of Barbados (Jaramillo and Burke, 1999). Carib grackles occasionally engage in dunking behaviour, the immersion of food items in water before consumption (Morand-Ferron et al., 2004; see Hickey, 2005 for a photograph). Previous field observations have revealed that dry food items elicit a higher frequency of dunking than fresh items, raising the possibility that dunking facilitates the consumption of food types that are hard to ingest. In the first part of this paper, we provide evidence that dunking accelerates food ingestion and is thus an advantageous processing behaviour in this population. In the second part, we test the effects of sex, age, and density of conspecifics on kleptoparasitism, as well as item profitability and loss of physical control over food. Finally, we examine whether potential hosts can respond flexibly to variation in the risk of kleptoparasitism and we describe two strategies employed by hosts to reduce their losses.

\section{Does dunking help grackles to process food?}

Dunking behaviour has been reported in more than 30 bird species in the wild (see Table 1 in Morand-Ferron et al., 2004). It 
has been suggested to serve at least four different potential functions: use of water to wash food (e.g. marabou storks washing dung beetles; Seibt and Wickler, 1978), soften hard items (e.g. house crow softening dry bread; Jones, 1979), drown live prey (e.g. Eurasian sparrowhawk drowning Eurasian jay; Weekley, 1997), or use of food as a sponge to transport water to nestlings (e.g. Brewer's blackbird; Koenig, 1985). In the first three cases, dunking is among the techniques that Parker and Gibson (1977) would classify as proto-tool use, in which the environmental feature that functions as a tool (water) is not detached from the substrate or held by the animal. Only in the last case would dunking be considered a true tool behaviour (Beck, 1980; Lefebvre et al., 2002), where the environmental feature is detached and manipulated by the user. In free-ranging Carib grackles, previous work suggests that dunking might ease the ingestion of items that are difficult to swallow (Morand-Ferron et al., 2004). If Carib grackles derive some food processing benefits from dunking items in water, we predict handling and consumption times to be shorter with dunked items than they are with dry ones.

\subsection{Material and methods}

This experiment was conducted at three different field sites in the parish of St. James, Barbados: (1) the paved terrace of Seabourne residence, located in the grounds of the Bellairs Research Institute of McGill University; (2) Folkestone Park, located to the immediate south of Bellairs; and (3) a public parking lot in Holetown, located ca. $1 \mathrm{~km}$ south of Folkestone.

At the beginning of each session, the observer (J.M.F.) placed ca. 100 dry dog food pellets $(0.7 \mathrm{~cm}$ in diameter, $21 \%$ protein, Atlantic Marketing, Barbados) in a clump on the ground $1 \mathrm{~m}$ from a naturally occurring puddle of water. Dry dog food pellets are readily eaten by grackles in captivity and in the wild, even though they are difficult to swallow and need to be broken in smaller pieces through repeated pecking (Morand-Ferron et al., 2004). Each time a bird took a food item, the observer (if not already watching another individual) noted the time this individual took: (1) to first deposit the item on the ground, in the water, or on a perch in a tree (travel time) and (2) to swallow the item completely (total time). The observer also noted the behaviour of the individual, using the following categories: eat dunked item, eat dry item while on the ground, eat dry item while perched in a tree. For this experiment, we excluded cases where the individual lost its item to a kleptoparasite or abandoned it without successfully feeding on it. We used two different measures of handling time: total time and consumption time (total time minus travel time). After log transformations, these data were normally distributed and we performed a one-way ANOVA and Tukey post-hoc comparisons to compare total time and consumption time of the three different behavioural categories observed. The results are similar for the two time measures and we thus present results for consumption time only, as travel time is comparatively short (mean travel time $\pm \mathrm{sd}$ is $12.0 \pm 12.1 \mathrm{~s}$ compared with $166.8 \pm 176.6 \mathrm{~s}$ for consumption time) and depends on the experimental placement of food and water in the case of dunking.

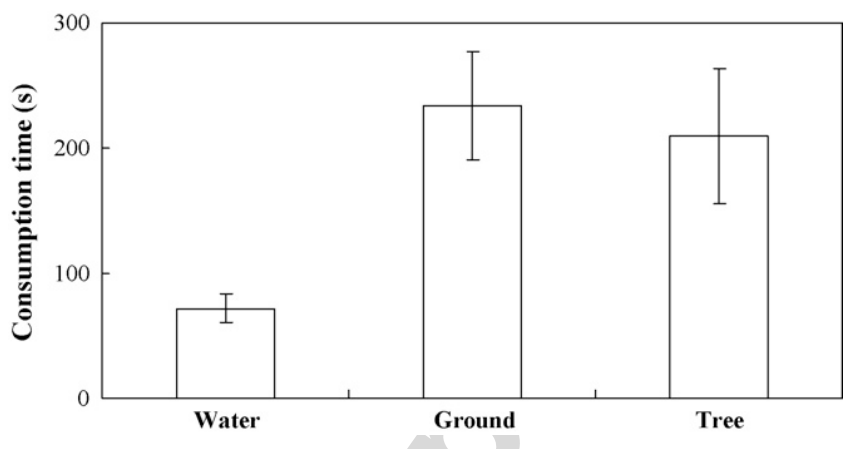

Fig. 1. Consumption time (mean \pm standard error) when dunking (water; $n=35$ ), depositing a dry item on the ground (ground; $n=29$ ), or on a perch (tree; $n=10$ ).

As not all birds observed in this experiment were marked (Carib grackles feed in open flocks with frequent changes in composition; see also Morand-Ferron et al., 2004), it is possible that some birds might have been observed more than once. We consider however that we reduced this possibility by performing observations at three different field sites, using individuals that had leg bands only once each (21 individually recognisable birds over a total of 74 observations), and switching between males and females and adults and juveniles whenever possible between observations in the same session (for a total of 20 sessions).

\subsection{Results}

We found a significant effect of the type of behaviour on consumption time (ANOVA: $F_{2,71}=20.4, p<0.001$; Fig. 1 ). Consumption time was two to three times shorter after dunking food in water than when consuming dry items on the ground (Tukey; $p<0.001$ ) or while perching in a tree (Tukey; $p=0.003$ ). There was no difference in consumption time when eating dry items on the ground or in a tree (Tukey; $p=0.99$ ).

These results suggest that one benefit of dunking is reduced handling time, thereby providing support for the food processing function of this behaviour in Carib grackles.

\section{Why are dunked items more often stolen than dry ones?}

In a previous study (Morand-Ferron et al., 2004), we observed that $13 \%$ of items that were dunked were lost to kleptoparasites, while only about $2 \%$ of items that were taken from the same food pile but not dunked were similarly lost. This difference could be due to many factors; from the results of the previous section, one could predict that, all other things being equal, kleptoparasites would preferentially target dunked items over dry ones, as dunked items require shorter handling times. In order to isolate the effect of item profitability from other variables, we compare the probability of kleptoparasitic attempts on dunked items versus dry items deposited on the ground at the same site. We predict more attempts on dunked than on dry items.

One reason dunking birds are more often victims of kleptoparasitism could be that they are manipulating food items at the water puddle, where there may be a greater build-up of birds (e.g. 
drinking and bathing) than in surrounding areas. Here, we compare conspecific density around focal birds handling a dunked item at the puddle versus birds manipulating a dunked item away from the puddle. We predict density to be higher at the water puddle, and to be significantly correlated with the probability of kleptoparasitic attempts and success.

The existence of two distinct dunking techniques in Carib grackles allows us to examine the effect of loss of physical control on food on the probability of kleptoparasitic attempts and success, while keeping item profitability constant. Grackles dunk by either releasing and retrieving an item from water (release technique) or by dipping an item in water while holding it in the bill (hold-while-dunking technique). We predict a higher probability of kleptoparasitic attempts and success on items dunked using the release technique compared to items held in the bill during dunking.

Sex and age biases in kleptoparasitic losses have been observed in many species, often with the subordinate sex or age class being preferentially robbed of food (e.g. Burger and Gochfeld, 1981; Ens et al., 1990). Assuming that males are dominant over females and adults over juveniles in Carib grackles (from what is known on other Quiscalus species; Johnson and Peer, 2001; Post et al., 1996), we predict a higher probability of kleptoparasitic attempts and success on females than on males and on juveniles than on adults.

To summarize, we predict more kleptoparasitism (1) at higher conspecific density, (2) on dunked items rather than dry items deposited on the ground (item profitability), (3) on items released in water than on items held in the bill while dunking (loss of physical control on food), (4) on females than on males, and (5) on juveniles than on adults.

\subsection{Material and methods}

The experiment was conducted on Seabourne terrace. Approximately, 100 food pellets (see Section 1) were placed in a clump on the ground $1 \mathrm{~m}$ from a naturally occurring puddle of water, modified to achieve a constant size $(50 \mathrm{~cm}$ in diameter) and water depth $(2 \mathrm{~cm})$ by replenishing it with tap water and lining it with plastic sheeting. The food pile and water puddle were replenished before each session and during a session if they were to be half-depleted. A similar experimental setting had already been in place for other field experiments in the 7 weeks preceding this study.

We performed opportunistic focal observations of grackles taking food pellets from the pile and manipulating them on the ground. All observations were recorded using a digital camcorder. An observer (M.V.) noted verbally on the videocassettes the number of times that each focal bird deposited a dry item on the ground, dunked an item in water through release and retrieval, dunked while holding the item in the bill, or deposited a dunked item on the ground. The observer also noted the sex and age category (adult or juvenile; Jaramillo and Burke, 1999) of the focal individual, the number of aggressive displays it performed, whether the bird was subject to kleptoparasitic attempts, and whether or not these attempts were successful. Kleptoparasitic acts consisted of rapid movements of a grackle towards a food item handled by a focal individual, which, when successful, ended with the kleptoparasite gaining possession of the item. A second observer (J.M.F.) doubled-checked the data while scoring videotapes and counted the number of grackles within a $15 \mathrm{~cm}$ radius of the focal individual for each act. We conducted 35 observational sessions of $30 \mathrm{~min}$ each, for a total of 4149 acts recorded.

As a grackle could engage in different behaviours with a single item, we had to analyse our data at the level of acts, not individuals or food items. Such data will not be statistically independent. To overcome this difficulty, we used generalised linear mixed models for autocorrelated data (\%GLIMMIX in SAS Version 8.2; Kuss, 2002) with binomial error and logit link, including day, session, and item as random nested factors. We examined the significance of one variable at a time in explaining the occurrence or absence of kleptoparasitic attempts (either missed or successful) and success. The null hypothesis was rejected when there was a significant deviation from chance; because we tested five hypotheses using the same data, we applied Bonferroni corrections throughout, setting the level for significance at 0.01 for each test (Stevens, 2002).

\subsection{Results}

We recorded a total of 4149 acts on 922 items manipulated on the experimental site by focal birds. Of those acts, $11.3 \%$ consisted in depositing a dry item on the ground $(n=470), 24.8 \%$ in depositing a dunked item on the ground $(n=1030), 50.7 \%$ in dunking an item using the release technique $(n=2104)$, and $13.1 \%$ in using the dunk hold technique $(n=545)$. The average rate of kleptoparasitic attempts (unsuccesful and successful) on all acts was $10.8 \%$, with $32.6 \%$ of these attempts ending in successful stealing events. Fig. 2 shows the proportion of unsuccessful and successful kleptoparasitic attempts on each of the four types of foraging behaviour.

\subsubsection{Conspecific density}

Grackles manipulating food at the water puddle were surrounded by a larger number of conspecifics than those handling

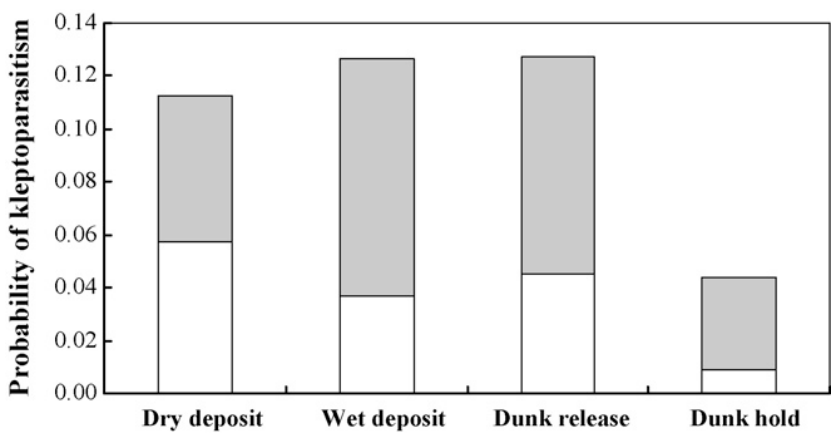

Fig. 2. Proportion of acts in which the focal bird lost its item to a kleptoparasite (in white) and received a missed kleptoparasitic attempt (in grey) for each type of behaviour recorded: deposit a dry item on the ground (dry deposit; $n=470$ ), deposit a dunked item on the ground (wet deposit; $n=1030$ ), dunk an item using the release technique $(n=2104)$, and dunk while holding an item in the bill $(n=545)$. 
a dunked item on a dry part of the site $(2.14 \pm 0.9$ versus $1.44 \pm 0.7$, respectively; GLIMMIX: $\left.F_{1,3217}=276.6, p<0.001\right)$. In general, the number of conspecifics within $15 \mathrm{~cm}$ of a focal individual was positively correlated with kleptoparasitic attempts (GLIMMIX: $F_{1,3583}=199.3, p<0.001$ ) and success (GLIMMIX: $\left.F_{1,3583}=84.9, p<0.001\right)$. In order to control for this confounding effect, we included grackle density counts in all subsequent analyses.

\subsubsection{Item profitability}

Contrary to our prediction, there were no significant differences in kleptoparasitic attempts or success on dry versus dunked items when both food types were deposited on the ground (11.3\% attempts on dry items versus $12.7 \%$ on dunked items: GLIMMIX: $F_{1,1062}=0.99, p=0.319 ; 5.7 \%$ successes on dry items versus $3.7 \%$ on dunked items: GLIMMIX: $F_{1,1062}=0.98$, $p=0.322)$. Once picked up, however, dry pellets were abandoned more often than dunked ones $(6.4 \%$ versus $0.1 \%$, respectively; Chi-square: $\left.\chi_{1}^{2}=352.5, p<0.001\right)$.

\subsubsection{Loss of physical control on food}

As predicted, attempts were more frequent on items released during dunking than on items held in the bill $(12.7 \%$ versus $4.4 \%$, respectively; GLIMMIX: $\left.F_{1,2288}=46.8, p<0.001\right)$; success rate was also higher for the former $(4.5 \%$ successes on items released in water versus $0.9 \%$ on items held in the bill: GLIMMIX: $\left.F_{1,2287}=26.5, p<0.001\right)$.

\subsubsection{Sex and age category}

Females and males were targeted and victimised equally often (attempts-GLIMMIX: $F_{1,3582}=0.96, p=0.327$; successesGLIMMIX: $F_{1,3582}=0.01, p=0.922$ ). We did not detect a significant difference between the probability of juveniles and adults being attacked (GLIMMIX: $F_{1,3582}=0.75, p=0.387$ ) and successfully robbed of food by kleptoparasites (GLIMMIX: $\left.F_{1,3582}=3.09, p=0.079\right)$. We obtained similar results when controlling for the behaviour performed by focal individuals (sex, attempts-GLIMMIX: $F_{1,3577}=0.86, p=0.353$; sex, successes-GLIMMIX: $F_{1,3579}=0.01, p=0.956$; age, attempts-GLIMMIX: $F_{1,3579}=1.60, p=0.206$; age, successes-GLIMMIX: $F_{1,3579}=3.92, p=0.048$ ).

To summarize, we observed more kleptoparasitism at higher conspecific density, and on items released in water than on items held in the bill while dunking, but found no significant effect of item profitability, sex or age of hosts (juveniles tended to be successfully robbed of food more often than adults, but this effect was not significant when applying Bonferroni's correction).

\section{How to reduce losses to kleptoparasites?}

In the previous section, we showed that dunking grackles could reduce their losses to conspecifics by holding food pellets in the bill while dunking. Paradoxically, grackles used the hold technique four times less frequently than the release technique (the latter accounted for $79.4 \%$ of dunks). If the safer, but less common hold-while-dunking technique involves additional costs with respect to the release technique, grackles should hold food items in the bill mostly when the risk of kleptoparasitism is high. We thus predict focal individuals to use the hold technique when surrounded by a larger number of conspecifics than when using the release technique.

When competing for food, both males and females Carib grackles sometimes engage in aggressive head-up displays, in which the bill is raised at an elevation of about $45^{\circ}$ and the grey nictitating membrane is drawn briefly over the eyes (Wiley, 1975). We predict grackles to engage in more numerous displays when they are the targets of more numerous kleptoparasitic attempts. We also predict that grackles using these displays will successfully reduce the risk of losing items to food thieves.

\subsection{Material and methods}

The prediction on dunking techniques was tested using the data and methods of Section 2. The predictions on displays were tested on the trials used in Section 2 and on a third set of field experiments conducted in very similar conditions, but with the camcorder zoomed in more closely on focal individuals. We pooled these trials with the ones analysed in the previous section (for a total of 3941 items) after we ascertained that there was no significant confounding effect of data source (GLIMMIX: $F_{1,3881}=1.19$, NS). We tested the effect of the number of displays per item dunked on the probability of kleptoparasitic attempts and success using generalised linear mixed models with binomial error and logit link, including data source, day and session as random nested factors, and conspecific density as a fixed effect.

\subsection{Results}

In comparison with the release technique, the hold-whiledunking technique was performed when focal birds were surrounded by a larger number of conspecifics (on average, $2.09 \pm 0.9$ conspecifics versus $2.28 \pm 1.0$, respectively; GLIMMIX: $F_{1,2289}=18.8, p<0.001$; Fig. 3 ). Including the hold technique in the dunking process significantly increased handling time compared with releasing items only $(52.4 \pm 23 \mathrm{~s}$ versus $44.8 \pm 21 \mathrm{~s}$, respectively; two-sample $t$-test: $t_{156}=-2.21$,

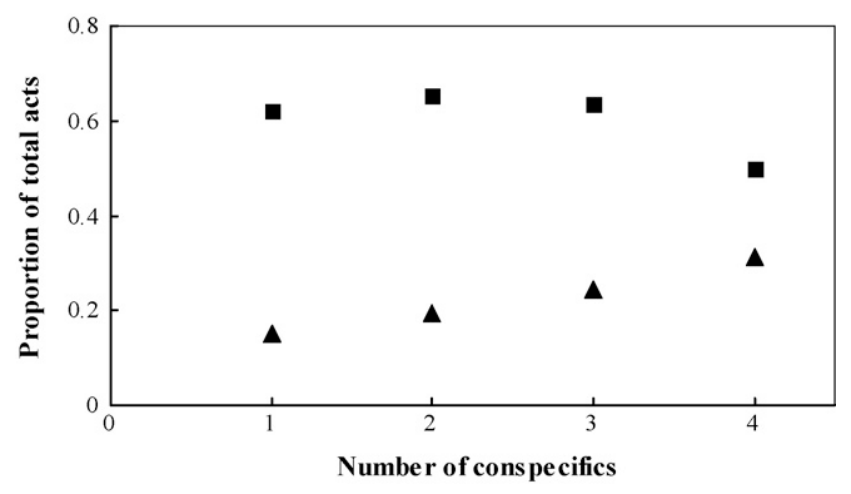

Fig. 3. Proportion of total acts performed at the study site that are dunk release (squares) and dunk hold (triangles) in function of the number of conspecifics located $15 \mathrm{~cm}$ or less from the focal bird. 
$p=0.028)$. The hold technique did not seem to be restricted to a small part of the population as 17 out of the $23(73.9 \%)$ banded birds observed dunking in this study engaged in both the release and hold-while-dunking technique, sometimes using the two techniques in manipulating a single item.

As predicted, focal individuals receiving more kleptoparasitic attacks engaged in more head-up displays per item (GLIMMIX: $F_{1,3881}=20.2, p<0.001$ ). Birds engaging in more displays per dunked item significantly reduced their probability of being robbed of food by kleptoparasites (GLIMMIX: $F_{1,3881}=7.3$, $p=0.007)$

These results suggest that holding items in the bill while dunking and engaging in head-up displays are efficient antikleptoparasitic tactics in Carib grackles.

\section{General discussion}

Our finding that dunked items could be ingested more quickly (including the time spent dunking) than dry ones is consistent with the idea that dunking is a food processing behaviour easing the ingestion of items that are difficult to swallow. Dunking is included in proto-tool behaviours by Parker and Gibson (1977), as the animal uses an element of the environment (water) to modify the characteristics of a resource (food). Animals making use of a proto- or a true tool may suffer greater kleptoparasitic losses compared with individuals engaged in other foraging behaviours on similar items, but there have been very few attempts to make this comparison in the field. In order to examine why dunking birds were robbed of food more often than non-dunking birds (Morand-Ferron et al., 2004), we tested five potential predictors of kleptoparasitism in free-ranging grackles. We found that dunking birds were more often kleptoparasitised because (1) they manipulated items at the water puddle where the density of conspecifics, and thus the risk of kleptoparasitism, was high and (2) they released items from the bill during handling. Birds eating dry items have the possibility of flying directly into trees where they usually perch without any conspecific in close proximity (Morand-Ferron, personal observation); dunking can thus be considered more risky as it involves manipulating the item at the water puddle.

We did not find an effect of sex or age of the host, nor of increased item profitability on kleptoparasitic occurrence. Surprisingly, kleptoparasites did not preferentially target dunked items over dry items, despite the observation that dunked items could be swallowed more quickly and that grackles seemed to value dry items less than dunked ones (dunked items were abandoned far less often than dry items). The most probable explanation for this lack of preference is that kleptoparasites could not distinguish visually whether an item has been previously dunked by the host or not. In another series of experiments, grackles presented with pre-soaked items sometimes walked to the puddle before swallowing the items without dunking, suggesting that the distinction between dry and soaked items was not made visually upon collection of the food, but later while the food was held in the bill (Lefebvre, unpublished obs.). Other bird species have been found to target preferentially items of higher energetic value when value could be easily assessed by the klep- toparasites (food type or item size; Brockmann and Barnard, 1979).

Many proto- or true tool use behaviours require releasing food items from the bill or hand during processing (e.g. fooddropping, use of a wedge, or of a hammer). In Carib grackles, loss of physical control on food was an important determinant of kleptoparasitic attacks and successes. Grackles handling dry food sometimes deposit it on the ground or on a perch, but they usually hold it between the toes after doing so (Morand-Ferron, personal observation). Dunking grackles most often let food items fall into water from a standing position and then lowered the head to retrieve it (release technique). Despite being very brief, this period of loss of control on food seemed to render dunking grackles vulnerable to kleptoparasitism. A similar situation has been observed in Japanese macaques, where individuals throwing wheat grains on the surface of water were often kleptoparasitised by conspecifics, but not those holding potatoes in the hand and rubbing them free of sand in water (Kawai, 1965). The release of items by hosts creates opportunities for stealthful kleptoparasitism, in which a thief can obtain an item while avoiding interaction with the host (Giraldeau and Caraco, 2000). This strategy might allow successful kleptoparasitism by individuals who would usually lose in aggressive contests over food items. For example, subordinate ravens have been observed engaging in cache raiding (stealth kleptoparasitism), but not in aggressive displacement (Bugnyar and Kortschal, 2002).

The rarity of the hold version of dunking behaviour in Carib grackles could be due to limited individual flexibility in the form of the behaviour exhibited. However, most dunking individuals $(73.9 \%)$ could use both techniques and some items were processed using a combination of the two techniques, which seems to rule out this possibility. An alternative explanation could be that the hold technique, despite reducing costs incurred through losses to food thieves, involves some other costs that the release technique does not confer. The increase in handling time associated with the use of the hold-while-dunking technique seems to support this explanation, but could also be due to other changes in the behaviour of birds dunking at high conspecific density (e.g. increased investment in conspecific monitoring, etc.). Other anti-kleptoparasitic behaviours have been shown to involve costs (Barnard and Stephens, 1981; Schenkeveld and Ydenberg, 1985; Vickery and Brooke, 1994; Stienen and Brenninkmeijer, 1999), thereby creating a trade-off between the risk of losing the item to kleptoparasites and the possible reduction in foraging rate through anti-theft action. Because they are often costly, protection behaviours must be performed flexibly, in accordance with variations in the risk of kleptoparasitism (Dally et al., 2006), which Carib grackles seemed to do by using the hold technique more often when conspecific density was high. Grackles also increased their use of aggressive head-up displays when subject to more kleptoparasitic attacks. This generally reduced food losses to kleptoparasites, but probably also involved costs in time, energy and risk of injury (mutual displays between a kleptoparasite and a potential host sometimes escalated into overt aggression; Morand-Ferron, unpublished observation). 
Overall, the probability of kleptoparasitic attempts and success on Carib grackles seemed to be more strongly determined by host behaviour than by characteristics of the host or of the item in its possession. Because of certain requirements of food processing behaviours, such as the need to release food items or to use a part of the environment where the density of competitors is high, food processing individuals might be particularly vulnerable to exploitation compared with individuals engaged in alternative foraging tactics. Comparative evidence suggests that tool and proto-tool use is more frequent in large-brained, innovative species (Lefebvre et al., 2002; Reader and Laland, 2002). It is thus possible that, in such taxa, potential hosts are behaviourally flexible enough to avoid "scrounging costs" by resorting to alternative foraging tactics or engaging in anti-kleptoparasitic behaviours when foraging under high risk of food theft.

\section{Acknowledgements}

We would like to thank Donald L. Kramer, Simon M. Reader, Neeltje Boogert, Sarah E. Overington, and two anonymous reviewers for helpful comments on a previous version of the paper. We are also grateful to Daniel Sol for his advice on statistical techniques. Funding for this work was provided by a Natural Sciences and Engineering Research Council of Canada (NSERC) grant to L.L. and a NSERC postgraduate scholarship to J.M.F. This study was conducted under McGill Animal Use Protocol number 4887 and complies with the laws of Barbados.

\section{References}

Barnard, C.J., Stephens, H., 1981. Prey size selection by lapwings in lapwing/gull associations. Behaviour 77, 1-22.

Beck, B.B., 1980. Animal Tool Behavior: The Use and Manufacture of Tools by Animals. Garland, New York, 307 pp.

Brockmann, H.J., Barnard, C.J., 1979. Kleptoparasitism in birds. Anim. Behav. 27, 487-514.

Bugnyar, T., Kortschal, K., 2002. Scrounging tactics in free-ranging ravens, Corvus corax. Ethology 108, 993-1009.

Burger, J., Gochfeld, M., 1981. Age-related differences in piracy behaviour of four species of gulls. Behaviour 77, 242-267.

Dally, J.M., Clayton, N.S., Emery, N.J., 2006. The behaviour and evolution of cache protection and pilferage. Anim. Behav. 72, 13-23.

Ens, B.J., Esselink, P., Zwarts, L., 1990. Kleptoparasitism as a problem of prey choice: a study on mudflat-feeding curlews, Numenius arquata. Anim. Behav. 39, 219-230.
Giraldeau, L.-A., Caraco, T., 2000. Social Foraging Theory. Princeton University Press, Princeton, 362 pp.

Goodwin, D., 1986. Crows of the World, second ed. British Museum, London, 299 pp.

Hickey, G., 2005. Dunking birds. Nat. Aust. Winter, 16.

Jackson, J., 1985. The dunking habit of common grackles. Am. Birds 39, 261-262.

Jaramillo, A., Burke, P., 1999. New World Blackbirds: The Icterids. Princeton University Press, Princeton, $431 \mathrm{pp}$.

Johnson, K., Peer, B.D., 2001. Great-tailed grackle. Birds N. Am. 576, 1-27.

Jones, C.G., 1979. Birds dunking food. Br. Birds 72, 189-190.

Kawai, M., 1965. Newly-acquired pre-cultural behavior of the natural troop of Japanese monkeys on Koshima islet. Primates 6, 1-30.

Koenig, W.D., 1985. Dunking of prey by Brewer's blackbirds: a novel source of water for nestlings. Condor 87, 444-445.

Kuss, O., 2002. How to use SAS for logistic regression with correlated data. SUGI 27, 261.

Lefebvre, L., Nicolakakis, N., Boire, D., 2002. Tools and brains in birds. Behaviour 139, 939-973.

Morand-Ferron, J., Lefebvre, L., Reader, S.M., Sol, D., Elvin, S., 2004. Dunking behaviour in Carib grackles. Anim. Behav. 68, 1267-1274.

Norris, K., Freeman, A., Vincent, J.F., 2000. The economics of getting high: decisions made by common gulls dropping cockles to open them. Behaviour $137,783-807$.

Parker, S.T., Gibson, K.R., 1977. Object manipulation, tool use and sensorimotor intelligence as feeding adaptations in Cebus monkeys and great apes. J. Human Evol. 6, 623-641.

Post, W., Poston, J.P., Bancroft, G.T., 1996. Boat-tailed grackle. Birds N. Am. 207, 1-19.

Reader, S.M., Laland, K.N., 2002. Social intelligence, innovation, and enhanced brain size in primates. Proc. Natl. Acad. Sci. U.S.A. 99, 44364441 .

Schenkeveld, L.E., Ydenberg, R.C., 1985. Synchronous diving by surf scoter flocks. Can. J. Zool. 63, 2516-2519.

Seibt, U., Wickler, W., 1978. Marabou storks wash dung beetles. Z. Tierpsychol. 46, 324-327.

Stevens, J.P., 2002. Applied Multivariate Statistics for the Social Sciences, fourth ed. Lawrence Erlbaum Associates, Mahwah, 699 pp.

Stienen, E.W.M., Brenninkmeijer, A., 1999. Keep the chicks moving: how sandwich terns can minimize kleptoparasitism by black-headed gulls. Anim. Behav. 57, 1135-1144.

Vickery, J.A., Brooke, M., 1994. The kleptoparasitic interactions between great frigatebirds and masked boobies on Henderson island, South Pacific. Condor 96, 331-340.

Visalberghi, E., Fragaszy, D.M., 1990. Food-washing behaviour in tufted capuchin monkeys, Cebus apella, and crabeating macaques, Macaca fascicularis. Anim. Behav. 40, 829-836.

Weekley, D.R., 1997. Eurasian sparrowhawk drowning Eurasian jay. Br. Birds 90, 524-526.

Wiley, R.H., 1975. Multidimensional variation in an avian display: implications for social communication. Science 190, 482-483. 